01

\title{
Оптимизация анодной мембраны с прострельной мишенью в системе источников мягкого рентгеновского излучения для проведения процессов рентгеновской нанолитографии
}

\author{
(С) П.Ю. Глаголев, ${ }^{1}$ Г.Д. Демин, ${ }^{1}$ Г.И. Орешкин, ${ }^{1}$ Н.И. Чхало, ${ }^{2}$ Н.А. Дюжев ${ }^{1}$ \\ ${ }^{1}$ Национальный исследовательский университет „МИЭТ“, \\ 124498 Зеленоград, Москва, Россия \\ ${ }^{2}$ Институт фоиики микроструктур РАН, \\ 603950 Нижний Новгород, Россия \\ e-mail: skirdovf@mail.ru
}

Поступило в Редакцию 16 апреля 2020 г.

В окончательной редакции 16 апреля 2020 г.

Принято к публикации 16 апреля 2020 г.

\begin{abstract}
Предложен способ оптимизации конструкции и состава анодной мембраны с прострельной мишенью в системе источников мягкого рентгеновского излучения на основе автоэмиссионных триодов, предназначенной для реализации задач в области рентгеновской нанолитографии. Данный способ позволяет предотвратить деградацию рабочих характеристик системы в случае возникновения сильной электростатической деформации анода под действием управляющего электрического поля в межэлектродном пространстве триодов. Для этой цели рассмотрено введение в конструкцию системы дополнительного управляющего электрода, позволяющего компенсировать деформацию анодной мембраны до приемлемого уровня и тем самым стабилизировать работу рентгеновских источников. Построена численная модель электростатического прогиба анодного узла в модифицированной конструкции, на основе которой определены оптимальный состав и геометрические параметры анодной мембраны с компенсирующим электродом. В частности, найдено оптимальное расстояние между анодной мембраной в начальном (недеформированном) состоянии и компенсирующим электродом (равное $5 \mu \mathrm{m}$ ), при котором к данным электродам следует приложить минимальную разницу напряжений (около $1.15 \mathrm{kV}$ ) для предотвращения критического прогиба мембраны $(0.72 \mu \mathrm{m}$ при радиусе мембраны $750 \mu \mathrm{m})$. Так же показано, что, в силу своей предельно высокой жесткости $(>80 \mathrm{GPa})$, алмазоподобные пленки являются наиболее перспективным материалом для анодного электрода. Полученные результаты также могут быть полезны для разработки миниатюрных устройств генерации рентгеновского излучения для различных применений.
\end{abstract}

Ключевые слова: рентгеновская нанолитография, микрофокусная рентгеновская трубка, прострельная мишень, матрица анодных узлов, электростатическая деформация.

DOI: $10.21883 / J T F .2020 .11 .49964 .137-20$

\section{Введение}

В настоящее время наблюдается стремительное возрождение одного из ключевых направлений физики - автоэмиссионной электроники, где за последние несколько лет были достигнуты впечатляющие результаты, как в области прорывных экспериментальных исследований [1-4], так и в отношении успешной реализации прототипов твердотельных электронных устройств на ее основе (диодов [5,6], транзисторов [7,8], наносенсоров [9], портативных рентгеновских трубок [10] и т.д.). Автоэмиссионная электроника посвящена разработке фундаментальных принципов и технологии построения электронных устройств в микро- и нанометровом диапазонах проектных норм, принцип действия которых основан на эффекте „холодной“ эмиссии электронов из твердотельной среды в вакуумный (либо квазивакуумный) проводящий канал под действием сильного электрического поля [11]. Поскольку баллистический транспорт электронов в вакуумном канале происхо- дит практически без соударений, технология вакуумной электроники обладает рядом преимуществ перед технологией полупроводниковых приборов: вакуумные микро- и наноэлектронные устройства могут работать на более высоких частотах (в суб-THz и THz-области), в более широком температурном диапазоне, а также в присутствии сильной радиации. В то же время работа полупроводниковых устройств зачастую оказывается нестабильной при таких экстремальных условиях, что ограничивает их применение в важнейших отраслях экономики (космос, ядерная энергетика, нефте- и газодобыча).

С другой стороны, актуальным вопросом современной наноэлектроники является обеспечение масштабирования электронных компонент к минимальным проектным нормам (вплоть до $10 \mathrm{~nm}$ и ниже), что требует применения новых литографических методов, обладающих высокой разрешающей способностью [12]. Одним из таких методов является безмасочная рентгеновская нанолитография, использующая длины волн рентгеновского 
излучения в диапазоне от 0.4 до $4 \mathrm{~nm}$ для формирования топологических рисунков с предельным разрешением в области десятков нанометров [13]. В отличие от своих конкурентов процесс рентгеновской нанолитографии не нуждается в применении дорогостоящих масок, обладает высоким быстродействием и позволяет формировать структуры с большим аспектным соотношением (до 100:1 [14]). Указанные преимущества могут быть достигнуты посредством использования системы миниатюрных рентгеновских источников на основе автоэмиссионных триодных структур, обладающей низким энергопотреблением и способной обеспечить быстрое сканирование (последовательное прохождение разверточных строк, на которые разбивается формируемый топологический рисунок) и безмасочное экспонирование образца рентгеновским лучом для моментального формирования на нем топологического рисунка [15]. Под образцом подразумевается полученная в ходе выполнения технологических операций (окисления, осаждения, напыления и проч.) структура (например, кремниевая пластина с перечнем функциональных слоев) с нанесенным на нее рентгенорезистом, из которой в процессе рентгеновской нанолитографии будут формироваться отдельные топологические элементы. Такие источники рентгеновского излучения крайне эффективны и для других сфер применения, таких как медицинская техника, оборудование для рентгенофлуоресцентного анализа, системы безопасности и проч.

Автоэмиссионные триодные структуры в составе рентгеновских источников позволяют создать направленный поток высокоэнергетичных электронов, стремительно пролетающих от катода к аноду с прострельной рентгеновской мишенью (менее чем за 1 ps при расстоянии „катод-анод“ в несколько десятков $\mu \mathrm{m}$ [16]). В свою очередь, размер электронного пучка регулируется под действием управляющего напряжения сеточного электрода, что обеспечивает получение фокусных пятен малого диаметра на прострельной мишени, что необходимо для генерации узконаправленных электромагнитных волн в рентгеновском диапазоне.

Ранее в [17-19] нами были рассмотрены концептуальные и технологические основы создания массива микрофокусных рентгеновских трубок на основе электронной системы из матрицы анодных узлов (перфорированная анодная мембрана с прострельной мишенью) и матрицы автоэмиссионных катодно-сеточных узлов (АКУ), представленных в виде кремниевых катодов острийного типа с набором управляющих сеточных электродов (рис. 1,a). В процессе моделирования конструкции электронной системы было выявлено, что при подаче на анод питающего напряжения, достаточного для возникновения автоэмиссионного тока, возникает электростатическая деформация анодной мембраны, что может отрицательно сказываться на широком спектре ключевых рабочих параметров рентгеновских источников - величине автоэмиссионного тока (приводить
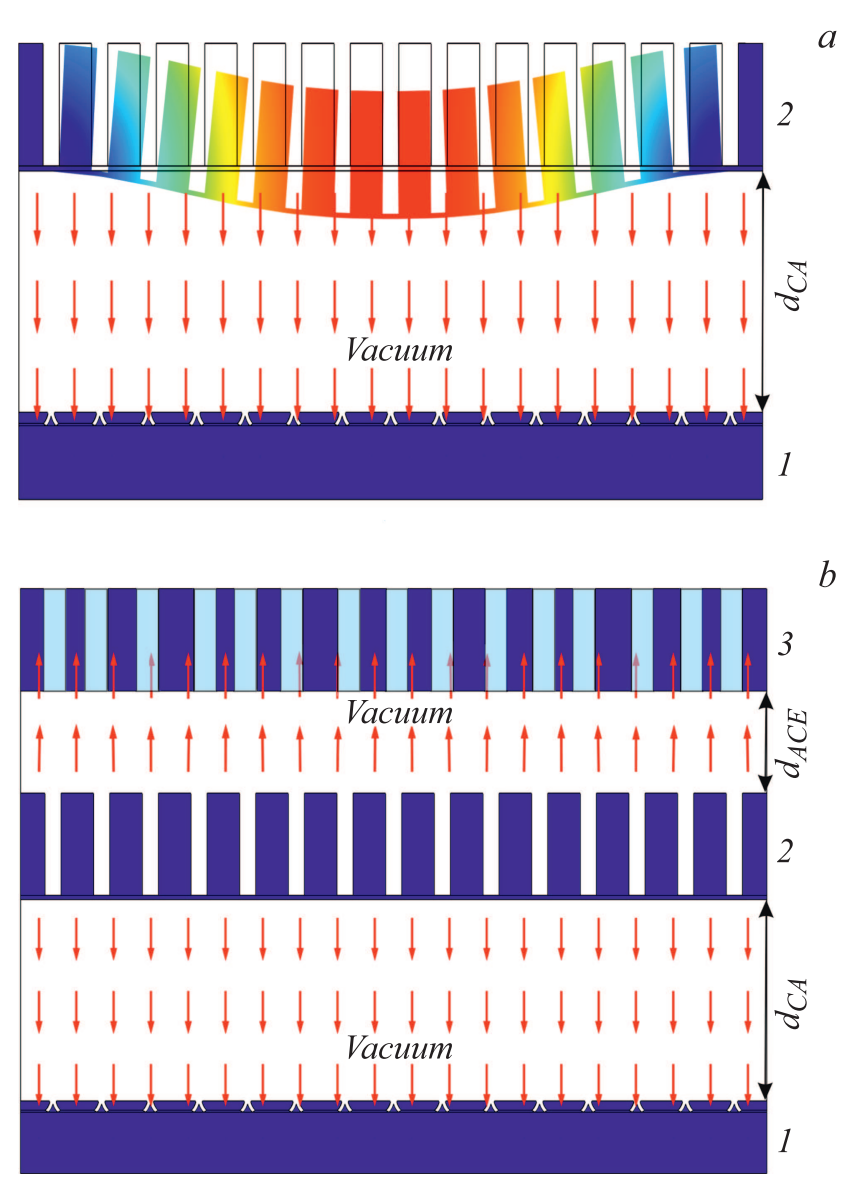

Рис. 1. Схематическое изображение системы источников мягкого рентгеновского излучения на основе матрицы автоэмиссионных катодных узлов (АКУ) и матрицы анодных узлов $(a)$ без компенсирующего электрода и $(b)$ с компенсирующим электродом. Матрица АКУ (1) состоит из набора кремниевых острийных катодов с управляющими сеточными электродами. Матрица анодных узлов (2) в начальной конструкции состоит из бериллиевой прострельной мишени, расположенной на перфорированной анодной мембране. Компенсирующий электрод (3) предназначен для минимизации электростатического прогиба матрицы анодных узлов. Стрелками обозначено направление вектора электрического поля.

к его флуктуациям), угловой направленности рентгеновского излучения и, как следствие, разрешающей способности топологического рисунка, формируемого методами безмасочной рентгеновской литографии [18]. Для устранения данного негативного эффекта в настоящей работе предложена модификация конструкции матрицы анодных узлов, состоящая в добавлении к ней дополнительного управляющего электрода на заданном расстоянии от прострельной мишени, электрическое поле от которого компенсирует деформацию анодной мембраны (рис. $1, b$ ). Предложенное решение позволяет стабилизировать работу системы источников мягкого рентгеновского излучения на основе автоэмиссионных триодов. 


\section{1. Оптимизация конструкции матрицы анодных узлов}

В изначальной конструкции система микрофокусных рентгеновских трубок включает в себя матрицу АКУ, матрицу анодных узлов и рентгенооптическую систему МЭМС зеркал [17]. Одиночный АКУ состоит из кремниевого нанокатода и шины сеточного электрода. Автоэмиссионный ток, создаваемый в процессе полевой эмиссии электронов с каждого элемента матрицы АКУ, контролируется запирающим напряжением на соответствующих шинах сеточных электродов. Матрица анодных узлов включает в себя рентгенопрозрачную бериллиевую пленку, в которой генерируется рентгеновское излучение в результате воздействия на пленку электронного пучка с автокатода, и анодную мембрану, предназначенную для создания электрического поля в межэлектродном пространстве между анодным узлом и АКУ. В процессе бондинга анодной и катодной части системы рентгеновских источников выходные рентгеновские окна матрицы анодных узлов самосовмещаются с катодно-сеточными узлами матрицы АКУ (располагаются напротив друг друга), в то время как ее конструкция обеспечивает теплоотвод с выходной прострельной мишени, что становится критичным при эмиссии электронов с высокой энергией (более $1 \mathrm{keV}$ ). В связи с этим важной задачей является выбор оптимального состава и конструкции матрицы анодных узлов для обеспечения быстрого оттока тепла в процессе генерации рентгеновского излучения. Более того, вследствие сильных электрических полей, необходимых для генерации автоэлектронной эмиссии (порядка нескольких $\mathrm{V} / \mathrm{nm}$ ), матрица анодных узлов подвергается значительной электростатической деформации (рис. $1, a$ ), что в значительной степени может препятствовать стабильной работе микрофокусных рентгеновских трубок в составе рентгеновского литографа. Важным фактором, влияющим на формирование направленности рентгеновского пучка (излучения), является пространственная ориентация рентгеновских окон в матрице анодных узлов. Предварительные оценки показывают, что максимальный прогиб $D_{m}^{\max }$ матрицы анодных узлов, определяющий отклонение рентгеновских окон от вертикального положения в деформированной мембране, не должен превышать критического значения $D_{M(t h)}^{\max }$, при котором уже становится проблематичным выровнять кривизну поля изображения на пластине с рентгенорезистом посредством оптической системы МЭМС-зеркал. Допустимой величиной кривизны можно считать величину, лежащую в пределах $\pm D_{o f} \approx \lambda / N_{A}^{2}$, где $D_{o f}$ - глубина фокуса проекционного объектива, $\lambda-$ рабочая длина волны. В предметной плоскости этой кривизне соответствует максимальное отклонение $\Delta= \pm D_{o f} M$, где $M$ - уменьшение объектива. Для модельной схемы литографа $N_{S A}=0.4, \lambda=11.4 \mathrm{~nm}$, $M=10$ [18], величина $D_{o f}=0.72 \mu \mathrm{m}$. Отсюда рассчитывается допустимое отклонение $D_{M(t h)}^{\max }$ анодной матрицы от плоскости, равное $\pm 0.72 \mu \mathrm{m}$ (максимальный размах высот составляет около $1.42 \mu \mathrm{m})$ для выбранного диаметра мембраны $(1.5 \mathrm{~mm})$.

Для решения указанных выше проблем в настоящей работе рассматривается оптимизация конструкции матрицы анодных узлов путем введения дополнительного управляющего электрода на заданном расстоянии от анодной мембраны, электрическое поле от которого способно скомпенсировать ее электростатический прогиб (рис. $1, b$ ). Для вывода рентгеновского излучения в компенсирующем электроде также предусмотрены рентгеновские окна большего диаметра, нежели чем в перфорированной анодной мембране, что связано с расхождением рентгеновского пучка после выхода из прострельной мишени. Для демонстрации эффективности предлагаемой концепции нами проведено численное моделирование конструкции матрицы анодных узлов с компенсирующим электродом с целью определения ее оптимальных параметров.

\section{2. Модель электростатической деформации матрицы анодных узлов}

В программном пакете COMSOL MultiPhysics [20], основанном на методе конечных элементов, была построена модель электростатической деформации матрицы анодных узлов как в случае отсутствия, так и в случае присутствия компенсирующего электрода в составе ее конструкции (рис. 1). Данный программный пакет был выбран вследствие наличия в нем физических модулей, необходимых для одновременного моделирования двух взаимосвязанных между собой физических процессов: 1) распределения электрического поля в межэлектродном пространстве (модуль „Electrostatics“) и 2) механической деформации анодной мембраны под действием рассчитанной электростатической силы, возникающей между матрицей анодных узлов и матрицей АКУ (модуль „Solid Mechanics“). Для корректного представления результатов была предусмотрена опция сглаживания расчетной сетки по Лапласу в процессе ее построения для случая деформируемой формы матрицы анодных узлов.

\section{3. Основные уравнения}

Электростатическое поле $\mathbf{E}=-\nabla V$ в вакуумном пространстве между матрицей анодных узлов и матрицей АКУ рассчитывается из численного решения уравнения Пуассона, представленного в дифференциальном виде

$$
-\nabla\left(\varepsilon_{m} \nabla V\right)=\frac{\rho_{T}^{m}}{\varepsilon_{0}},
$$

где $\varepsilon_{0}-$ электрическая постоянная, $\varepsilon_{m}$, - диэлектрическая проницаемость $m$ среды, $\rho_{V}^{m}-$ объемная плотность заряда в $m$ среде. В вакууме полагается, что $\rho_{V}^{v a c}=0$, 
$\varepsilon_{m}=1$. В качестве начальных условий задается потенциал $V=V_{A}$ на матрице анодных узлов и нулевой потенциал $(V=0)$ на матрице АКУ (в приближении, что напряжение сеточного электрода (порядка нескольких десятков V) не оказывает сильного воздействия на деформацию анодной мембраны).

Значение поля $\mathbf{E}=\mathbf{E}_{A}$ на поверхности анодной мембраны необходимо для расчета вектора и деформации матрицы анодных узлов, возникающей под действием электростатической силы $\mathbf{f}_{e}$, что соответствует уравнению

$$
\nabla(F S)^{T}+\mathbf{F}_{g V}=0
$$

где $F=\nabla \mathbf{u}+I, I-$ единичный тензор, $\mathbf{F}_{g V}-$ внешняя сила гравитации, действующая на единицу объема анодной мембраны. Следует отметить, что сила гравитации не оказывает заметного эффекта на прогиб мембраны, возникающий под действием электростатической силы в межэлектродном пространстве. По этой причине величиной $\mathbf{F}_{g V}$ в расчетах деформации матрицы анодных узлов можно пренебречь. Второй тензор напряжений ПиолыКирхгофа определяется следующей формулой:

$$
S=2 \frac{\partial H_{e m e}}{\partial C},
$$

где $C=F^{T} F-$ тензор упругости 4-го ранга, а электрическая энтальпия $H_{e m e}$ (термодинамический потенциал) в пренебрежении электрической поляризацией диэлектриков и эффектом электрострикции материалов будет выражаться как

$$
H_{e m e}=W_{S}(C)-0.5 \varepsilon_{0} J C^{-1}:\left(\mathbf{E}_{A} \otimes \mathbf{E}_{A}\right),
$$

где $J=\operatorname{det}(F), W_{S}(C)=0.5 \varepsilon_{e l}:\left(C: \varepsilon_{e l}+2 \sigma_{0}\right)=0.5 \varepsilon_{e l}$ : $\left(\sigma+\sigma_{0}\right)$ - плотность энергии упругой деформации (для случая рассмотрения линейных упругих материалов), $\varepsilon_{e l}=\varepsilon-=\varepsilon_{\text {inel }}, \quad \varepsilon-$ тензор общей деформации материала, $\varepsilon_{i n e l}-$ тензор неупругой деформации, $\sigma$ - электромеханический тензор напряжений, $\varepsilon_{0}-$ электромеханический тензор начальных или внешних напряжений. Для упрощения записи приведенных соотношений оператор „:“ обозначает двойное тензорное произведение.

Будем также полагать, что тензор напряжений является непрерывным на границе между материалами (граничные условия)

$$
\mathbf{n}_{i}\left(\sigma_{i+1}-\sigma_{i}\right)=0
$$

где $\mathbf{n}_{i}-$ вектор нормали к границе $i$-го материала, $\sigma_{i(i+1)}$ - тензор напряжений в $i(i+1)$-ом материале. В вакуумном пространстве (в отсутствие магнитных полей) данный тензор описывается как $\sigma_{v a c}=\varepsilon_{0} \mathbf{E} \otimes \mathbf{E}-0.5\left(\varepsilon_{0} \mathbf{E} \cdot \mathbf{E}\right) I$, причем $(\mathbf{E} \otimes \mathbf{E})_{i j}=E_{i} E_{j}$.

В свою очередь, для оценки коэффициента $\beta_{C}$ усиления электрического поля (отношение максимальной напряженности электрического поля на поверхности катода к напряженности поля между плоскими электродами) на острийном катоде мы использовали выражение вида

$$
\beta_{C}=E_{C}^{\max } \frac{d_{C A}}{V_{A}},
$$

где $E_{C}^{\max }$ - максимальное поле на поверхности катода (локальное электрическое поле на его вершине), $d_{C A}-$ расстояние между матрицей АКУ и матрицей анодных узлов в недеформированном состоянии (рис. 1). Данный коэффициент позволяет судить о степени изменения величины электрического поля $E_{C}^{\max }$ на вершине катода с расстоянием $d_{C A}$, что важно для оценки вариации величины автоэмиссионного тока с каждого элемента АКУ при заданном электростатическом прогибе мембраны.

\section{4. Результаты моделирования}

Проведенное нами моделирование позволяет определить оптимальные физические и конструктивные параметры матрицы анодных узлов с компенсирующим электродом, включение которого необходимо для устранения нежелательной деформации анодной мембраны с прострельной мишенью в процессе автоэлектронной эмиссии. В качестве материала анодной мембраны изначально выбран углерод (diamond), причем диаметр мембраны в составе матрицы анодных узлов был принят равным $1.5 \mathrm{~mm}$. Следует отметить, что в процессе моделирования толщина анодного узла $\left(h_{A}=10 \mu \mathrm{m}\right)$ и толщина компенсирующего электрода $\left(h_{C E}=50 \mu \mathrm{m}\right)$ оставались неизменными, а величина напряжения на аноде была изначально зафиксирована на уровне $C_{A}=2 \mathrm{kV}$, достаточном для поддержания стабильного автоэмиссионного тока. В качестве варьируемых геометрических параметров были выбраны следующие величины расстояние $d_{C A}$ между матрицей АКУ и матрицей анодных узлов в первоначальном (недеформированном) состоянии анодной мембраны, расстояние $D_{A C E}$ между анодной мембраной и компенсирующим электродом в первоначальном (недеформированном) состоянии анодной мембраны (рис. 1).

На рис. 2, а проиллюстрирована зависимость коэффициента $\beta_{C}$ усиления электрического поля на вершине острийного катода от расстояния между $d_{C A}$ матрицей АКУ и недеформированной матрицей анодных узлов. Как видно из рисунка, данная зависимость имеет нелинейный характер, что связано с нелинейным уменьшением максимального поля $E_{C}^{\max }$ с ростом $d_{C A}$, что легко видеть из рис. $2, b$. В свою очередь, согласно формуле (5), сам коэффициент $\beta_{C}$ плавно увеличивается в диапазоне от 43.1 до 46.5 при вариации расстояния от 3 до $20 \mu \mathrm{m}$. Рис. $2, b$, показывает, что поле $E_{C}^{\max }$ практически линейно возрастает с ростом рабочего напряжения $V_{A}$ на аноде (от 1 до $2 \mathrm{kV}$ ) на больших расстояниях (10 и $20 \mu \mathrm{m})$, в то время как с приближением матрицы анодных узлов к катоду острийного типа в области больших напряжений появляется небольшой нелинейный участок 

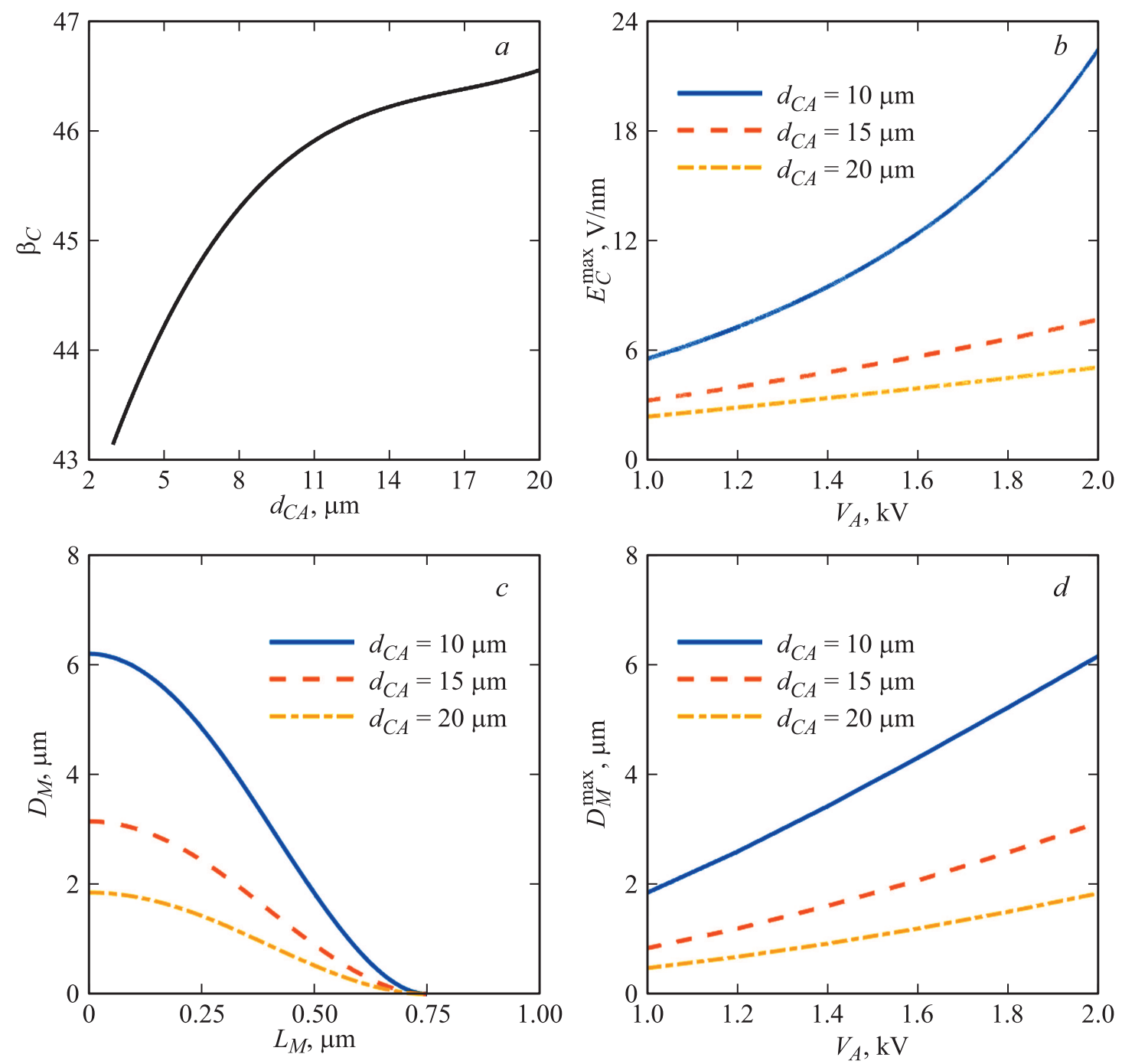

Pис. 2. $a-$ коэффициент усиления электрического поля на наконечнике острийного катода $\beta_{C}$ как функция от расстояния „матрица АКУ-матрица анодных узлов“ $d_{C A}$. Напряжение на аноде $V_{A}=2 \mathrm{kV}$. $b$ - максимальная амплитуда электрического поля $R E_{C}^{\max }$ на наконечнике острийного катода как функция от напряжения $V_{A}$ на анодной мембране в случае вариации $d_{C A}$. $c$ - электростатический прогиб анодной мембраны $D_{M}$ вдоль линии $L_{M}$ для различных расстояний $d_{C A}$ (при напряжении $V_{A}$, равном $2 \mathrm{kV}$ ), где линия $L_{M}-$ радиальная линия, проложенная от центра мембраны к ее краю. $d-$ максимальное смещение $D_{M}^{\max }$ анодной мембраны как функция от напряжения $V_{A}$ на анодной мембране в случае вариации $d_{C A}$.

зависимости. В заданном диапазоне электрических полей должна наблюдаться стабильная полевая эмиссия электронов (максимальное значение поля $E_{C}^{\max }$ на поверхности кремниевого нанокатода превышает пороговое поле автоэмиссии $E_{t h}^{F E}-$ около $\left.2 \mathrm{~V} / \mathrm{nm}[21]\right)$. Также нами проведена оценка электростатической деформации матрицы анодных узлов в отсутствие компенсирующего электрода. На рис. 2, $c$ показан электростатический прогиб $D_{M}$ анодной мембраны вдоль радиальной линии $L_{M}$ на ее поверхности при напряжении на аноде, равном $2 \mathrm{kV}$. Данный прогиб заметно превышает допустимый предел деформации $D_{M(t h)}^{\max }$, равный $0.72 \mu \mathrm{m}$ для заданного диаметра мембраны $(1.5 \mathrm{~mm})$, при котором не возникают изменение угловой направленности рентгеновского излучения и искажение разрешающей способности рентгеновского нанолитографа в рабочем диапазоне напряжений. Максимальная электростатическая деформация $D_{M}^{\max }$ матрицы анодных узлов при этом практически линейно возрастает с напряжением $V_{A}$, что следует из рис. $2, d$. Следует отметить, что расстояние $d_{C A}$ варьировалось от 10 до $20 \mu \mathrm{m}$. Включение компенсирующего электрода с управляющим напряжением $V_{C E}$ в состав конструкции матрицы анодных узлов приводит к заметному снижению ее деформации (практически в несколько раз), что видно из картины трехмерного распределения деформаций по поверхности анодной мембраны, представленной на рис. 3. Рисунок приведен для случая, когда разница напряжений между компенсирующим электродом и анодной мембраной $V_{C E}-V_{A}=2 \mathrm{kV}$, расстояние $D_{C A}=10 \mu \mathrm{m}, d_{A C E}=10 \mu \mathrm{m}$. На рис. $4, a$ 
Matrix of anode nodes (without a compensating electrode)

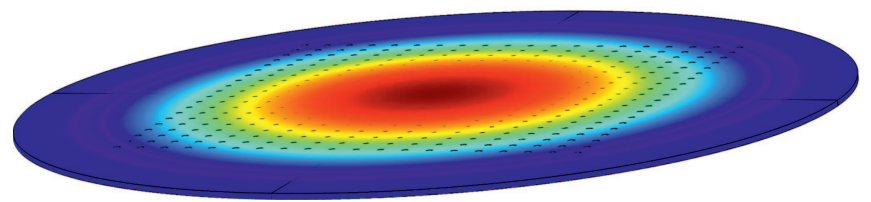

Matrix of anode nodes (with a compensating electrode)
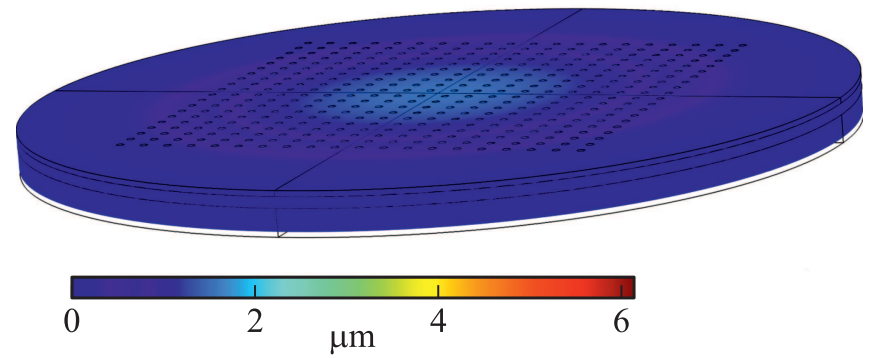

Рис. 3. Трехмерное распределение электростатической деформации матрицы анодных узлов $(a)$ в присутствии и $(b)$ в отсутствие компенсирующего электрода для случая, когда $V_{C E}-V_{A}=2 \mathrm{kV}, d_{C A}=10 \mu \mathrm{m}, d_{A C E}=10 \mu \mathrm{m}$.

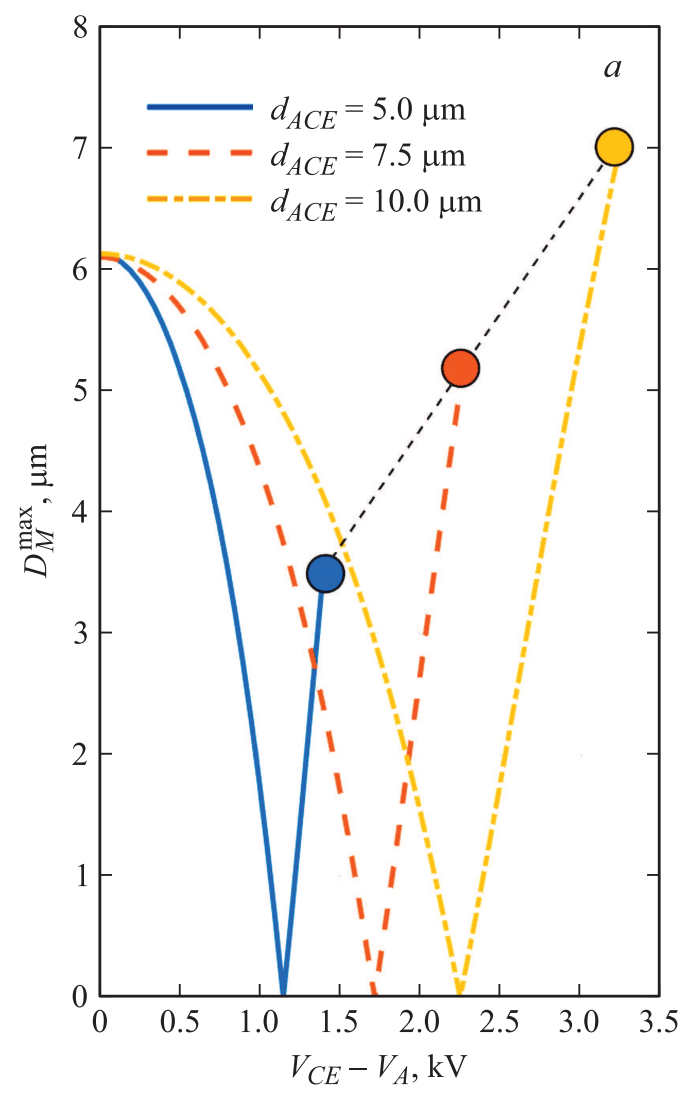

представлена максимальная электростатическая деформация анодной мембраны $D_{M}^{\max }$ в присутствии компенсирующего электрода в зависимости от разности напряжений на компенсирующем электроде $V_{C E}$ и на матрице анодных узлов $V_{A}$ для различных расстояний $d_{A C E}$ (от 5 до $10 \mu \mathrm{m}$ ). Минимальная (близкая к нулю) деформация матрицы анодных узлов наблюдалась в случае пороговых значений $\left(V_{C E}-V_{A}\right)_{t h}$, равных 1.15 , 1.65 и $2.25 \mathrm{kV}$ для соответствующих расстояний $d_{A C E}-$ $5,7.5$ и $10 \mu \mathrm{m}$. В случае, когда $V_{C E}-V_{A}>\left(V_{C E}-V_{A}\right)_{t h}$, наблюдается деформация матрицы анодных узлов в обратную сторону - по направлению к компенсирующему электроду. В связи с этой особенностью кривые на рис. 4, а ограничены тонкой пунктирной линией, пересечение с которой соответствует случаю, когда прогиб матрицы анодных узлов составляет $70 \%$ от расстояния $d_{A C E}$. Данная граница взята с запасом и соответствует величине электрического поля $E_{A C E}^{\max }$ в межэлектродном пространстве „,анод-компенсирующий электрод“, равной $1 \mathrm{~V} / \mathrm{nm}$, что составляет $50 \%$ от значения порогового поля $E_{t h}^{F E}$, при котором может начаться эмиссия электронов с поверхности анода на компенсирующий электрод, а также возможен нежелательный контакт и

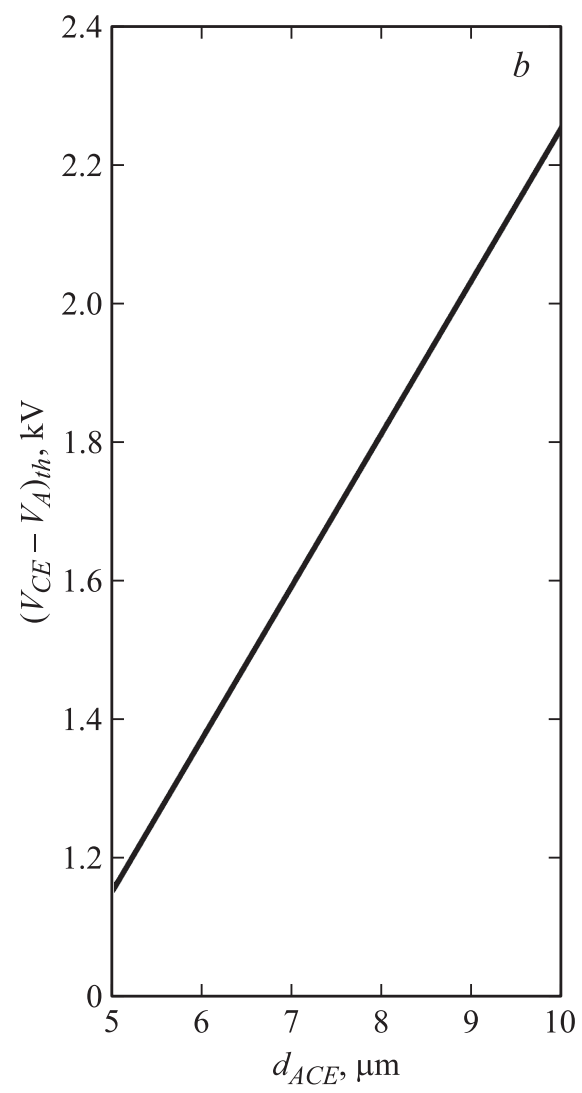

Pис. 4. $a-$ максимальный электростатический прогиб $D_{M}^{\max }$ матрицы анодных узлов как функция от напряжения $V_{C E}-V_{A}$ при различных расстояниях $d_{A C E}$ между компенсирующим электродом и анодной мембраной. На пересечении кривых с тонкой пунктирной линией прогиб матрицы в сторону компенсирующего электрода составляет $70 \%$ от расстояния $d_{A C E}$ (обозначено круглыми точками на графике). $b-$ пороговое значение разности напряжений $\left(V_{C E}-V_{A}\right)_{t h}$ между компенсирующим электродом и анодной мембраной, при котором электростатический прогиб матрицы анодных узлов близок к нулю, в зависимости от расстояния $d_{A C E}$. Расстояние $d_{C A}$ в обоих случаях полагается равным $10 \mu \mathrm{m}$. 


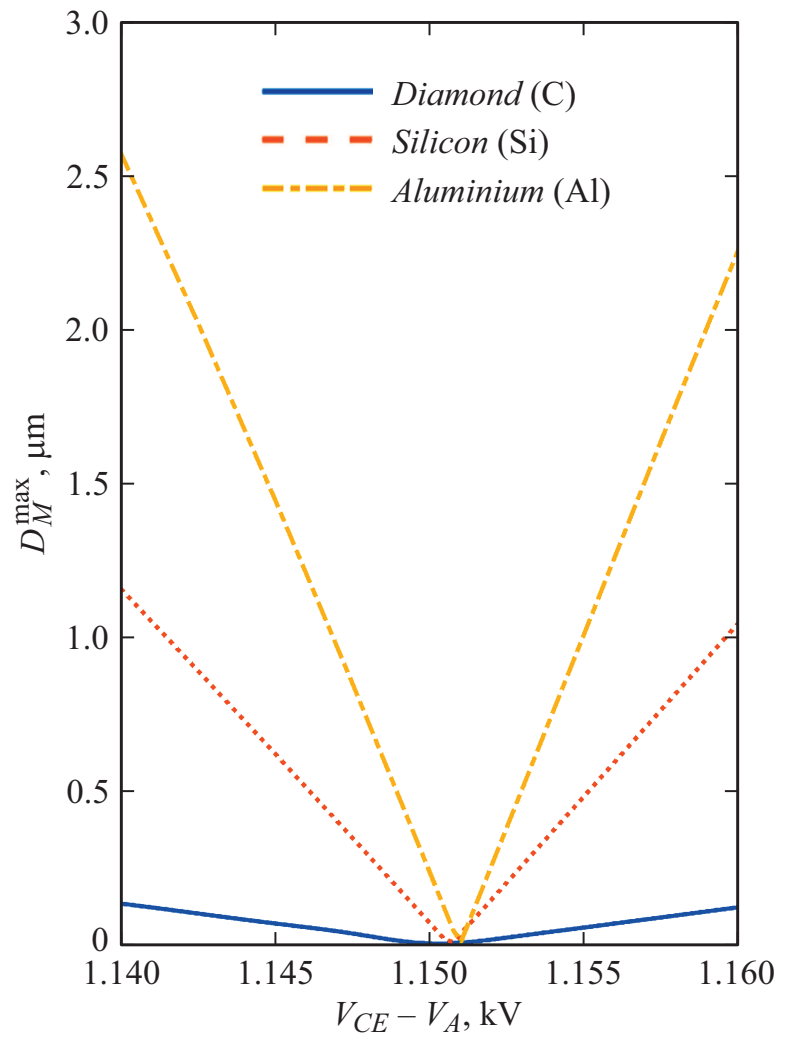

Рис. 5. Сравнение максимального электростатического прогиба $D_{M}^{\max }$ матрицы анодных узлов (с компенсирующим электродом) для различных материалов анодной мембраны как функция от разности напряжений на компенсирующем электроде $V_{C E}$ и анодной мембране $V_{A}$. Расстояние $d_{A C E}$ (,анодная мембрана-компенсирующий электрод“) равно $5 \mu \mathrm{m}$, расстояние $d_{C A}$ („матрица АКУ-матрица анодных узлов“) равно $10 \mu \mathrm{m}$ соответственно. Напряжение $V_{A}$ принято равным $2 \mathrm{kV}$.

слипание электродов за счет электростатических сил. При этом существует линейная зависимость между величиной межэлектродного расстояния $d_{A C E}$ и пороговой разностью напряжений $\left(V_{C E}-V_{A}\right)_{t h}$, при которой деформация матрицы анодных узлов становится минимальной (рис. 4,b). Следует отметить, что при указанных значениях $\left(V_{C E}-V_{A}\right)_{t h}$ не достигается поле, превышающее значение $E_{t h}^{F E}$, при котором может возникнуть нежелательный эффект эмиссии электронов в пространстве между анодной мембраной и компенсирующим электродом.

Также была исследована вариация максимума $D_{M}^{\max }$ электростатической деформации матрицы анодных узлов для различных материалов анодной мембраны и компенсирующего электрода при фиксированном расстоянии $d_{A C E}=5 \mu \mathrm{m}$. Данный анализ проводился с целью выявления оптимального состава анодной матрицы, позволяющего обеспечить стабильную работу микрофокусного рентгеновского источника даже в случае возникновения случайных отклонений разницы напряжений $V_{C E}-V_{A}$ от своего порогового значения $\left(V_{C E}-V_{A}\right)_{t h}$, при котором деформация мембраны становится минимальной. В качестве материала анодной мембраны и компенсирующего электрода были рассмотрены кремний $(\mathrm{Si})$, алюминий $(\mathrm{Al})$ и алмазоподобные $(\mathrm{C})$ пленки. На рис. 5 представлены соответствующие зависимости $D_{M}^{\max }$ от разности напряжений на электродах для приведенных выше материалов. Как видно из рисунка, алюминий и кремний демонстрируют наибольшую крутизну нарастания деформации в области напряжений от 1.14 до $1.16 \mathrm{kV}$ (вблизи порогового значения $1.15 \mathrm{kV}$ ), что, в конечном счете, может привести к искажению направленности рентгеновского излучения, а также вариации эмиссионного тока в пространстве между матрицей АКУ и матрицей анодных узлов. По этой причине алмазоподобные пленки, обладающие жесткостью более $80 \mathrm{GPa}$ [22] и меньшей (по сравнению с рассмотренными нами материалами) вариацией величины прогиба при отклонении разности напряжений $V_{C E}-V_{A}$ от своего порогового значения $\left(V_{C E}-V_{A}\right)_{t h}$, представляются наиболее привлекательными для предотвращения случайной электростатической деформации матрицы анодных узлов. Стоит также отметить, что алмаз обладает высокой теплопроводностью (в диапазоне от 2000 до $2500 \mathrm{~W} /(\mathrm{m} \cdot \mathrm{K})$ при температуре $300 \mathrm{~K}[23])$, что позволяет предотвратить сильный разогрев матрицы анодных узлов автоэмиссионным током и тем самым увеличивает срок службы анодной мембраны на основе алмазоподобных пленок.

\section{Заключение}

Таким образом, в работе рассмотрен один из способов оптимизации матрицы анодных узлов в составе микрофокусного рентгеновского источника, направленный на минимизацию ее электростатической деформации при протекании автоэмиссионного тока. Для этой цели нами предлагается введение в конструкцию матрицы дополнительного управляющего электрода на заданном расстоянии от поверхности анодной мембраны, что открывает путь к подстройке и компенсации ее электростатического прогиба. В качестве материалов компенсирующего электрода и электрода анодной мембраны были рассмотрены кремний, алюминий и алмазоподобные пленки. Из моделирования следует, что наиболее подходящим материалом для указанных электродов являются алмазоподобные пленки, поскольку по сравнению с другими материалами они менее подвержены электростатическому прогибу в силу своей предельно высокой жесткости (более $80 \mathrm{GPa}$ [22]). По этой причине электроды, выполненные из данного материала, демонстрируют устойчивость к отклонению разности напряжений $V_{C E}-V_{A}$ между компенсирующим электродом и матрицей анодных узлов от пороговой величины $\left(V_{C E}-V_{A}\right)_{t h}$, при которой деформация матрицы становится минимальной. Для заданных межэлектродных расстояний $d_{A C E}$, варьируемых в диапазоне от 5 до $10 \mu \mathrm{m}$, определен набор значений 
$\left(V_{C E}-V_{A}\right)_{t h}$. Выбранный диапазон расстояний является оптимальным, так как в случае, когда $d_{A C E}<5 \mu \mathrm{m}$, случайная флуктуация напряжения в области порогового значения $\left(V_{C E}-V_{A}\right)_{t h}$ может стать причиной слипания электродов, а при расстоянии $d_{A C E}>10 \mu \mathrm{m}$ на компенсирующий электрод необходимо подавать напряжение свыше $4 \mathrm{kV}$, что приводит к сильному разогреву анодной мембраны. Выявлена линейная зависимость между пороговой разницей напряжений $\left(V_{C E}-V_{A}\right)_{t h}$ и расстоянием $d_{A C E}$ между анодной мембраной и компенсирующим электродом. Из данной зависимости следует, что минимальная разница напряжений $\left(V_{C E}-V_{A}\right)_{t h}=1.15 \mathrm{kV}$ достигается при оптимальном расстоянии $d_{A C E}=5 \mu \mathrm{m}$ (когда межэлектродное расстояние $d_{A C E}=10 \mu \mathrm{m}$ ). Полученные результаты в значительной степени облегчают решение проблемы возникновения электростатического прогиба матрицы анодных узлов в составе источников мягкого рентгеновского излучения, что способствует повышению стабильности и надежности процессов рентгеновской нанолитографии на их основе.

\section{Финансирование работы}

Работа выполнена с использованием оборудования Центра коллективного пользования „Микросистемная техника и электронная компонентная база“ Национального исследовательского университета „Московский институт электронной техники“, поддержанного Минобрнауки России, при финансовой поддержке Гранта президента РФ (№ 075-15-2019-1139).

\section{Конфликт интересов}

Авторы заявляют, что у них нет конфликта интересов.

\section{Список литературы}

[1] Han J.-W., Seol M.-L., Moon D.-I., Hunter G., Meyyappan M. // Nat. Electron. 2019. Vol. 2. P. 405. DOI: $10.1038 / \mathrm{s} 41928-019-0289-\mathrm{z}$

[2] Liu M., Li T., Wang Y. // J. Vac. Sci. Technol. B. 2017. Vol. 35. P. 031801. DOI: $10.1116 / 1.4979049$

[3] Huang Y., Deng Z., Wang W., Liang C., She J., Deng S., $X u$ N. // Sci. Rep. 2015. Vol. 5. P. 10631. DOI: $10.1038 /$ srep10631

[4] Guerrera S.A., Akinwande A.I. // Nanotech. 2016. Vol. 27. P. 295302. DOI: 10.1088/0957-4484/27/29/295302

[5] Zhang P., Lau Y.Y. // J. Plasma Phys. 2016. Vol. 82. P. 595820505. DOI: 10.1017/S002237781600091X

[6] Chang W.-T., Hsu H.-J., Pao P.-H. // Micromachines. 2019. Vol. 10. P. 858. DOI: $10.3390 / \mathrm{mi} 10120858$

[7] Han J.-W., Moon D.-I., Meyyappan M. // Nano Lett. 2017. Vol. 17. P. 2146. DOI: 10.1021 /acs.nanolett.6b04363

[8] Xu J., Gu Z., Yang W., Wang Q., Zhang X. // Nanoscale Res. Lett. 2018. Vol. 13. P. 311. DOI: 10.1186/s11671-018-2736-6

[9] Liu M., Lei Y., Yang Y., Li T., Wang Y. // Proc. 2019 Intern. Conf. Manipulation, Automation and Robotics at Small Scales. Helsinki, Finland, 2019. Vol. 1.

DOI: $10.1109 /$ marss.2019.8860991
[10] Djuzhev N.A., Demin G.D., Gryazneva T.A., Kireev V.Yu., Novikov D.V. // Proc. 2018 IEEE Conf. of Russian Young Researchers in Electrical and Electronic Engineering IEEE, Moscow, Russia, 2018. 1974. DOI: 10.1109/EIConRus.2018.8317498

[11] Egorov N., Sheshin E. (ed.) Field Emission Electronics. Springer Series in Advanced Microelectronics, Vol. 60. Springer International Publishing, 2017. 568 p.

[12] Sebastian E.M., Jain S.K., Purohit R., Dhakad S.K., Rana R.S. // Mater. Today: Proc. 2020. in press. DOI: $10.1016 /$ j.matpr.2020.02.505

[13] Chkhalo N.I., Lopatin A.Ya., Pestov A.E., Salashchenko N.N., Demin G.D., Dyuzhev N.A., Makhiboroda M.A. // Proc. SPIE International Conference on Micro- and Nano-Electronics 2018, Zvenigorod, Russian Federation, 2018, 110221M. DOI: $10.1117 / 12.2522105$

[14] Xue C., Zhao J., Wu Y., Yu H., Yang S., Wang L., Zhao W., Wu Q., Zhu Z., Liu B., Zhang X., Zhou W., Tai R. // Appl. Surf. Sci. 2017. Vol. 425. P. 553-557. DOI: $10.1016 /$ j.apsusc.2017.07.010

[15] Djuzhev N.A., Demin G.D., Gryazneva T.A., Pestov A.E., Salashchenko N.N., Chkhalo N.I., Pudonin F.A. // Bull. Lebedev Phys. Inst. 2018. Vol. 45. N 1. C. 1. DOI: $10.3103 / \mathrm{S} 1068335618010013$

[16] Татаренко Н.И., Кравченко В.Ф. Автоэмиссионные наноструктуры и приборы на их основе. М.: Физматлит, 2006. $192 \mathrm{c}$

[17] Дюжев Н.А., Демин Г.Д., Филиппов Н.А., Евсиков И.Д., Глаголев П.Ю., Махиборода М.А., Чхало Н.И., Салащенко Н.Н., Филиппов С.В., Колосько А.Г., Попов Е.О., Беспалов В.А. // ЖТФ. 2019. Т. 89. Вып. 12. C. 1836-1842. [Djuzhev N.A., Demin G.D., Filippov N.A., Evsikov I.D., Glagolev P.Y., Makhiboroda M.A., Chkhalo N.I., Salashchenko N.N., Filippov S.V., Kolosko A.G., Popov E.O., Bespalov V.A. // Tech. Phys. 2019. Vol. 64. N 12. P. 1742. DOI: 10.1134/S1063784219120053]

[18] Salashchenko N.N., Chkhalo N.I., Djuzhev N.A. // J. Surf. Invest.: X-Ray, Synchrotron Neutron Tech. 2018. Vol. 12. P. 944. DOI: $10.1134 / \mathrm{S} 1027451018050324$

[19] Demin G.D., Djuzhev N.A., Filippov N.A., Glagolev P.Yu., Evsikov I.D., Patyukov N.N. // J. Vac. Sci. Technol. B. 2019. Vol. 37. P. 022903. DOI: $10.1116 / 1.5068688$

[20] COMSOL Multiphysics v. 5.5, COMSOL AB, Stockholm, Sweden, https://www.comsol.com/

[21] Ding M., Sha G., Akinwande A.I. // IEEE Trans. Electron. Devices. 2002. Vol. 49. P. 2333. DOI: 10.1109/TED.2002.805230

[22] Hatada R., Flege S., Ashraf M.N., Timmermann A., Schmid C., Ensinger $W$. // Coatings. 2020. Vol. 10. P. 360. DOI: 10.3390/coatings10040360

[23] Shinde S.L., Goela J. // High Thermal Conductivity Materials. Springer, 2006. ISBN: 9780387251004 